BULLETIN OF THE

AMERICAN MATHEMATICAL SOCIETY

Volume 80, Number 3, May 1974

\title{
INDUCTIVELY DEFINED SETS OF REALS
}

\author{
BY DOUGLAS CENZER
}

Communicated by Alistair H. Lachlan, October 8, 1973

1. Introduction. The concept of inductive definability has become of great interest to recursion theorists in recent years. Recursion over natural numbers, ordinals, and higher type objects may itself be defined by an inductive operator-see for example [7] and [9]. Many results have been obtained characterizing the closures of inductive operators over the natural numbers, and relating lengths of inductive definitions to various interesting ordinals; see [3] for a brief summary.

The purpose of this note is to present results on the closure ordinals and sets of inductive operators over the continuum. Details will appear later in [2], [4], and [5].

2. Basic definitions and notation. An inductive operator $\Gamma$ over a set $X$ is a map from $P(X)$ to $P(X)$ such that for all $A, A \subseteq \Gamma(A)$. $\Gamma$ determines a transfinite sequence $\left\{\Gamma^{\sigma}: \sigma \in \mathrm{ORD}\right\}$, where $\Gamma^{\sigma}=U\left\{\Gamma^{\tau}: \tau<\sigma\right\}$ for $\sigma=0$ or $\sigma$ a limit and $\Gamma^{\sigma+1}=\Gamma\left(\Gamma^{\sigma}\right) . \Gamma$ is monotone if, for all $A, B$ in $P(X), A \subseteq B$ implies $\Gamma(A) \subseteq \Gamma(B)$. $\Gamma$ is positive if its application to a set $A$ involves only the positive part of $\chi_{A}$ (the characteristic function of $A$ ).

The closure ordinal $|\Gamma|$ of $\Gamma$ is the least ordinal $\sigma$ such that $\Gamma^{\sigma+1}=\Gamma^{\sigma}$; clearly $|\Gamma|$ always has cardinality less than or equal to $\operatorname{card}(X)$. The closure $\Gamma$ of $\Gamma$ is $\Gamma^{|\Gamma|}$, the set inductively defined by $\Gamma$.

For a class $C$ of inductive operators, the closure ordinal $|C|=\sup \{|\Gamma|$ : $\Gamma \in C\}$ and the closure algebra $\bar{C}=\{A: A$ is $1-1$ reducible to $\Gamma$ for some $\Gamma$ in $C$ \}. We write $C$-mon for the class of monotone operators in $C$.

In studying inductive operators over the continuum, we follow the usual convention that a real number is a function from the set $\omega$ of natural numbers to itself; thus the real line is ${ }^{\omega} \omega$.

3. Main results. The central result of our research is the following theorem.

THEOREM 1. (a) $\mid \Pi_{1}^{0}$-mon $|=| \Pi_{1}^{1}$-mon $|=| \Sigma_{1}^{1}$-mon $\mid=\aleph_{1}$;

(b) $\left(\Pi_{1}^{0} \text {-mon }\right)^{-}=\left(\Pi_{1}^{1} \text {-mon }\right)^{-}=\Pi_{1}^{1}$;

(c) $\left(\Sigma_{1}^{1} \text {-mon }\right)^{-}=\Sigma_{2}^{1}$.

AMS (MOS) subject classifications (1970). Primary 02F29; Secondary 02F27, 02F35, $02 \mathrm{~K} 30$.

Key words and phrases. Inductive definition, continuum, ordinal, closure, inductively parametrized. 
One direction of these equalities is derived from the existence of a $\Pi_{1}^{0}$ monotone operator $\Gamma$ with $|\Gamma|=\aleph_{1}$ and $\Gamma$ equal to the set of codes for well-orderings.

The other direction depends heavily on first proving that both $\Pi_{1}^{1}$ and $\Sigma_{1}^{1}$ monotone operators can be put in positiveform. See [1] for some related results.

Concerning nonmonotone inductive definitions, we obtain the following result by considering the essential countability of any computation on real numbers.

THEOREM 2. (a) $\left|\Sigma_{1}^{1}\right|=\aleph_{1}$;

(b) $\left(\Delta_{1}^{1}\right) \subseteq \Sigma_{2}^{1}$.

It is interesting to compare these two theorems with the corresponding results for inductive definitions over the natural numbers, where

$$
\mid \Pi_{1}^{0} \text {-mon }|=| \Pi_{1}^{1} \text {-mon }\left|=\omega_{1}<\right| \Delta_{1}^{1} \mid\langle| \Sigma_{1}^{1} \text {-mon } \mid
$$

and

$$
\left(\Pi_{1}^{0} \text {-mon }\right)^{-}=\left(\Pi_{1}^{1} \text {-mon }\right)^{-}=\Pi_{1}^{1} \varsubsetneqq\left(\Sigma_{1}^{1} \text {-mon }\right)^{-} \varsubsetneqq \Sigma_{2}^{1} .
$$

The simplest type of inductive definition over the reals would be one in which the real variable appeared only as a parameter. For example, if $R$ is a $\Pi_{1}^{0}$ relation such that for all $\alpha, \Gamma_{\alpha}$, defined by $n \in \Gamma_{\alpha}(A) \leftrightarrow R(n, \alpha, A)$, is an inductive operator over $\omega$, then there is an obvious $\Pi_{1}^{0}$ operator $\Gamma$ such that for all $n$ and $\alpha, n \in\left(\Gamma_{\alpha}\right)^{-}$iff $\langle n, \alpha\rangle \in \Gamma$. A similar fact holds if $\Pi_{1}^{0}$ is replaced by any other definability class.

We see by Theorem 1(b) and remark (2) that for $\Pi_{1}^{0}$ this process can be reversed: If $\Gamma$ is a $\Pi_{1}^{0}$ monotone operator over ${ }^{\omega} \omega$, then there is a $\Pi_{1}^{0}$ relation $R$ such that for all $\alpha, \Gamma_{\alpha}$, defined by $n \in \Gamma_{\alpha}(A) \leftrightarrow R(n, \alpha, A)$, is a monotone operator over $\omega$ and $\alpha \in \Gamma$ iff $0 \in \Gamma_{\alpha}$. We say that the $\Gamma_{\alpha}$ parametrize $\Gamma$.

Comparing Theorem 1(c) with remark (2), we see that this cannot be done for $\Sigma_{1}^{1}$ operators over ${ }^{\omega} \omega$. The surprising result here will be the extent of the definability class needed to parametrize $\Delta_{1}^{0}$ nonmonotone operators.

In general, we say that $A \subseteq{ }^{\omega} \omega$ can be inductively parametrized in $C$ if there is a $\left\{\Gamma_{\alpha}: \alpha \in{ }^{\omega} \omega\right\}$ in $C$ and a recursive $F$ such that for all $\alpha$ in ${ }^{\omega} \omega$, $\alpha \in A$ iff $F(\alpha) \in \Gamma_{\alpha}$. Let $\mathrm{PCl}(C)$ be the class of subsets of ${ }^{\omega} \omega$ which can be inductively parametrized in $C$.

By the above remarks, $\operatorname{PCl}\left(\Pi_{1}^{0}\right)=\Pi_{1}^{1}=\left(\Pi_{1}^{0}-\mathrm{mon}\right)^{-}$. The power of recursive inductive operators over the continuum can be seen in the following result.

THEOREM 3. (a) $\left|\Delta_{1}^{0}\right|=\aleph_{1}$;

(b) $\operatorname{PCl}\left(\Pi_{1}^{1}\right) \cup \operatorname{PCl}\left(\Sigma_{1}^{1}\right) \subseteq\left(\Delta_{1}^{0}\right)^{-}$. 
Inductive definitions of length greater than $\aleph_{1}$ are closely related to the functionals ${ }^{3} E$ and ${ }^{3} E^{\#}$ - see [8] for definitions. Let $\kappa_{1}$ be the ordinal of ${ }^{3} E, \kappa_{2}$ that of ${ }^{3} E^{\#} ; \kappa_{1}$ is less than $\kappa_{2}$. The following two results are implicit in [6].

Proposition 4. $\left|\Pi_{1}^{1}\right|=\kappa_{1} ;\left(\Pi_{1}^{1}\right)^{-}=$semirecursive in ${ }^{3} E$ (hyperanalytic).

Proposition 5. For all $n>1, \mid \Delta_{n}^{1}$-mon $|=| \Sigma_{2}^{1} \mid=\kappa_{2}$ and $\left(\Delta_{n}^{1}\right.$-mon) ${ }^{-}=$ $\left(\Sigma_{2}^{1}\right)^{-}=$semirecursive in ${ }^{3} E^{\#}$ (hyperprojective).

We can add the following.

THEOREM 6. $\left|\Pi_{2}^{1}\right|>\kappa_{2}$.

Hyperprojective inductive definitions can be analyzed in the manner of [3] and [10]. For example, we obtain the following analogue of a theorem of Richter from [10].

THEOREM 7. $\left|\left[\Delta_{2}^{1}, \Delta_{2}^{1}\right]\right|$ is the least ordinal which is recursively Mahlo in ${ }^{3} E^{\#}$ and parameters from ${ }^{\omega} \omega$.

All of the results in this paper are easily relativized with respect to a real parameter and have obvious boldface analogues.

\section{REFERENCES}

1. A. Blass and D. Cenzer, Cores of $\Pi_{1}^{1}$ sets of reals (to appear).

2. D. Cenzer, Monotone inductive definitions over the continuum, Notices Amer. Math. Soc. 21 (1974), A-19.

3. - Ordinal recursion and inductive definitions, J. Fenstad and P. Hinman (Ed.), Generalized Recursion Theory, Oslo, 1972, North-Holland, Amsterdam (to appear).

4. - Ordinal recursion over the continuum (to appear).

5. - Parametric inductive definitions and recursive operators over the continuum (to appear).

6. T. Grilliot, Hierarchies based on objects of finite type, J. Symbolic Logic 34 (1969), 177-182. MR 44 \#75.

7. P. Hinman, Recursion-theoretic hierarchies (to appear).

8. P. Hinman and Y. Moschovakis, Computability over the continuum, Logic Colloquium '69, North-Holland, Amsterdam, 1971, pp. 77-105.

9. S. C. Kleene, Recursive functionals and quantifiers of finite types. II, Trans. Amer. Math. Soc. 108 (1963), 106-142. MR 27 \#3521.

10. W. Richter, Recursively Mahlo ordinals and inductive definitions, Logic Colloquium '69 (Proc. Summer School and Colloq., Manchester, 1969), North-Holland, Amsterdam, 1971, pp. 273-288. MR 43 \#7331.

Department of Mathematics, University of Florida, Gainesville, Florida 32601 